Yukawa Institute Kyoto

YITP-01-34

hep-th/0105164

May 2001

\title{
Universal Lax pairs for Spin Calogero-Moser Models and Spin Exchange Models
}

\author{
V. I. Inozemtsev' and R. Sasaki \\ Yukawa Institute for Theoretical Physics \\ Kyoto University, Kyoto 606-8502, Japan
}

\begin{abstract}
For any root system $\Delta$ and an irreducible representation $\mathcal{R}$ of the reflection (Weyl) group $G_{\Delta}$ generated by $\Delta$, a spin Calogero-Moser model can be defined for each of the potentials: rational, hyperbolic, trigonometric and elliptic. For each member $\mu$ of $\mathcal{R}$, to be called a "site", we associate a vector space $\mathbf{V}_{\mu}$ whose element is called a "spin". Its dynamical variables are the canonical coordinates $\left\{q_{j}, p_{j}\right\}$ of a particle in $\mathbf{R}^{r},(r=$ rank of $\Delta)$, and spin exchange operators $\left\{\hat{\mathcal{P}}_{\rho}\right\}(\rho \in \Delta)$ which exchange the spins at the sites $\mu$ and $s_{\rho}(\mu)$. Here $s_{\rho}$ is the reflection generated by $\rho$. For each $\Delta$ and $\mathcal{R}$ a spin exchange model can be defined. The Hamiltonian of a spin exchange model is a linear combination of the spin exchange operators only. It is obtained by "freezing" the canonical variables at the equilibrium point of the corresponding classical CalogeroMoser model. For $\Delta=A_{r}$ and $\mathcal{R}=$ vector representation it reduces to the well-known Haldane-Shastry model. Universal Lax pair operators for both spin Calogero-Moser models and spin exchange models are presented which enable us to construct as many conserved quantities as the number of sites for degenerate potentials.
\end{abstract}

\footnotetext{
${ }^{1}$ permanent address: BLTP JINR, 141980 Dubna, Moscow Region, Russia
} 


\section{Introduction}

The essential part of our knowledge of quantum many-body systems is concerned with integrable models in one dimension. Among them, the Calogero-Moser models [1, 2, 3, 4] with long-range interactions are most popular during last decade. Their links to the models of solid-state physics [5, 6, 7, 8, 9, 10, 11, 13] have been found, and they are based on the possibility to introduce also the spin exchange interaction in a translation-invariant form. However, the CM models can be formulated n classical and quantum mechanics for any root system [14, 15, 16, 17, 18, 19], and one can guess that introduction of spin exchange can be done at least for some root systems too. There were several attempts [8, 9, 10, 12 in this direction, but they were far from being universal in a way for introducing spin into the CM models.

In this paper, we consider the possibility of unifying all the previous approaches to spin Calogero-Moser models and related models of spin exchange interactions obtained by "freezing" the canonical variables at the equilibrium points of the corresponding classical CM systems. This can be done by constructing universal Lax representations for degenerate forms of the CM potentials. There are also some indications that the corresponding models with most general elliptic potentials are also integrable [6, 13], but the construction of Lax pair in this case does not lead directly to integrability.

The organization of the paper is as follows. In Section 2, the universal Lax operators for the CM models with degenerate potentials [17, 18] is briefly recapitulated. The way of introducing spin exchange in the framework of the above formalism is proposed in Section 3 so as to prove the integrability of the spin CM models for all root systems. The existence of conserved quantities is guaranteed by the "sum to zero" condition for the second Lax operator. Section 4 is devoted to the models with spin exchange operators only. The corresponding Lax operators lead in the trigonometric case and $A_{r}$ root system to Haldane-Shastry model [5]. The Polychronakos model [8, 12] corresponds in this approach to the rational case with a confining $q^{2}$ potential. The final section is devoted to summary and comments. 


\section{Universal Lax Operator for Calogero-Moser Model with Degenerate Potential}

In this section we briefly recapitulate the essence of Calogero-Moser models based on any root system $\Delta$ (applicable to the exceptional and non-crystallographic root system) and the associated universal Lax pair formalism along with appropriate notation [16, 17, 18, 19] and background [14, 15] for the main body of this paper. Those who are familiar with the universal Lax pair formulation may skip this section and return when necessity arises. A Calogero-Moser model is a Hamiltonian system associated with a root system $\Delta$ of rank $r$, which is a set of vectors in $\mathbf{R}^{r}$ with its standard inner product, invariant under reflections in the hyperplane perpendicular to each vector in $\Delta$. In other words,

$$
s_{\alpha}(\beta) \in \Delta, \quad \forall \alpha, \beta \in \Delta, \quad s_{\alpha}(\beta)=\beta-\left(\alpha^{\vee} \cdot \beta\right) \alpha, \quad \alpha^{\vee} \equiv 2 \alpha /|\alpha|^{2} .
$$

The set of reflections $\left\{s_{\alpha}, \alpha \in \Delta\right\}$ generates a group $G_{\Delta}$, a finite reflection group, known as the Coxeter (Weyl) group. The set of roots $\Delta$ is decomposed into a disjoint sum of the positive roots $\Delta_{+}$and negative roots $\Delta_{-}$. The dynamical variables of the Calogero-Moser model are the coordinates $\left\{q_{j}\right\}$ and their canonically conjugate momenta $\left\{p_{j}\right\}$, which will be denoted by vectors in $\mathbf{R}^{r}$ with the standard inner product:

$$
q=\left(q_{1}, \ldots, q_{r}\right), \quad p=\left(p_{1}, \ldots, p_{r}\right), \quad p^{2}=p \cdot p=\sum_{j=1}^{r} p_{j}^{2} .
$$

The Hamiltonian for classical Calogero-Moser model with a degenerate potential reads:

$$
\mathcal{H}_{C}=\frac{1}{2} p^{2}+\frac{1}{2} \sum_{\rho \in \Delta_{+}} g_{|\rho|}^{2}|\rho|^{2} V(\rho \cdot q),
$$

in which the potential function $V$ is listed in the following Table 1:

\begin{tabular}{|l|c|c|c|}
\hline & $V(u)$ & $x(u)$ & $y(u)$ \\
\hline rational & $1 / u^{2}$ & $1 / u$ & $-1 / u^{2}$ \\
\hline trigonometric & $1 / \sin ^{2} u$ & $\cot u$ & $-1 / \sin ^{2} u$ \\
\hline hyperbolic & $1 / \sinh ^{2} u$ & $\operatorname{coth} u$ & $-1 / \sinh ^{2} u$ \\
\hline
\end{tabular}

Table 1: Functions appearing in the Hamiltonian and Lax pair.

Here we have omitted the scale factor for the trigonometric (hyperbolic) potential, for simplicity. The associated universal Lax pair operators read

$$
\begin{aligned}
L & =p \cdot \hat{H}+X, \quad X=i \sum_{\rho \in \Delta_{+}} g_{|\rho|}(\rho \cdot \hat{H}) x(\rho \cdot q) \hat{s}_{\rho} \\
\widetilde{M} & =\frac{i}{2} \sum_{\rho \in \Delta_{+}} g_{|\rho|}|\rho|^{2} y(\rho \cdot q) \hat{s}_{\rho}
\end{aligned}
$$


in which the functions $x(u)$ and $y(u)$ are listed in the Table 1. These functions are related by

$$
y(u) \equiv d x(u) / d u, \quad V(u)=-y(u)=x^{2}(u)+\text { constant }
$$

The real positive coupling constants $g_{|\rho|}$ are defined on orbits of the corresponding reflection group, i.e. they are identical for roots in the same orbit. That is, for the simple Lie algebra cases one coupling constant $g_{|\rho|}=g$ for all roots in simply-laced models and two independent coupling constants, $g_{|\rho|}=g_{L}$ for long roots and $g_{|\rho|}=g_{S}$ for short roots in non-simply laced models. The operators $\hat{H}_{j}$ and $\hat{s}_{\rho}$ obey the following commutation relations

$$
\begin{array}{r}
{\left[\hat{H}_{j}, \hat{H}_{k}\right]=0,} \\
{\left[\hat{H}_{j}, \hat{s}_{\alpha}\right]=\alpha_{j}\left(\alpha^{\vee} \cdot \hat{H}\right) \hat{s}_{\alpha},} \\
\hat{s}_{\alpha} \hat{s}_{\beta} \hat{s}_{\alpha}=\hat{s}_{s_{\alpha}(\beta)}, \quad \hat{s}_{\alpha}^{2}=1, \quad \hat{s}_{-\alpha}=\hat{s}_{\alpha} .
\end{array}
$$

In terms of these commutation relations it is easy to show that the canonical equations of motion can be represented in an operator form:

$$
\dot{q}_{j}=p_{j}, \quad \dot{p}_{j}=-\frac{\partial \mathcal{H}_{C}}{\partial q_{j}} \Longleftrightarrow \frac{d}{d t} L=[L, \widetilde{M}] .
$$

Let us choose an irreducible $D$-dimensional representation of the reflection (Weyl) group $G_{\Delta}$ to be denoted by $\mathcal{R}$. It is a collection of $\mathbf{R}^{r}$ vectors, to be called a "site", which form a single Weyl orbit:

$$
\mathcal{R}=\left\{\mu^{(1)}, \ldots, \mu^{(D)} \mid \mu^{(k)} \in \mathbf{R}^{r}\right\}
$$

That is any site of $\mathcal{R}$ can be obtained from any other site by the action of the reflection (Weyl) group. Thus the (length) ${ }^{2}$ of the vectors $\mu^{(k)}$ are equal:

$$
\left(\mu^{(j)}\right)^{2}=\left(\mu^{(k)}\right)^{2}, \quad \forall \mu^{(j)}, \mu^{(k)} \in \mathcal{R} .
$$

Then $L$ and $\widetilde{M}$ are $D \times D$ matrices whose elements are given by

$$
\left(\hat{H}_{j}\right)_{\mu \nu}=\mu_{j} \delta_{\mu \nu}, \quad\left(\hat{s}_{\rho}\right)_{\mu \nu}=\delta_{\mu, s_{\rho}(\nu)}=\delta_{\nu, s_{\rho}(\mu)} .
$$

The essence of the Lax pair is the following set of identities among the functions $\{x(\rho \cdot q)\}$ and $\{y(\rho \cdot q)\}$ expressed in matrix forms:

$$
[X, \widetilde{M}]=-\hat{H} \cdot \frac{\partial \mathcal{V}}{\partial q}, \quad \mathcal{V}=\frac{1}{2} \sum_{\rho \in \Delta_{+}} g_{|\rho|}^{2}|\rho|^{2} V(\rho \cdot q)
$$




$$
[p \cdot \hat{H}, \widetilde{M}]=i\left[-\frac{1}{2} \frac{\partial^{2}}{\partial q^{2}}, X\right]
$$

in which the right hand side of (2.14) is a diagonal matrix. The matrix $\widetilde{M}$ has a special property (see (2.36) of 18 ):

$$
\sum_{\mu \in \mathcal{R}} \widetilde{M}_{\mu \nu}=\sum_{\nu \in \mathcal{R}} \widetilde{M}_{\mu \nu}=-i \mathcal{V}_{S}, \quad \mathcal{V}_{S}=\frac{1}{2} \sum_{\rho \in \Delta_{+}} g_{|\rho|}|\rho|^{2} V(\rho \cdot q),
$$

in which $\mathcal{V}_{S}$ is independent of $\mu$ and $\nu$. Note that $\mathcal{V}_{S}$ is different from $\mathcal{V}$ in (2.14), which is quadratic in the coupling $g_{|\rho|}$, whereas $\mathcal{V}_{S}$ is linear. We can define a new matrix $M$,

$$
M=\widetilde{M}+i \mathcal{V}_{S} \times I, \quad I: \text { Identity operator, }
$$

which satisfies sum up to zero condition

$$
\sum_{\mu \in \mathcal{R}} M_{\mu \nu}=\sum_{\nu \in \mathcal{R}} M_{\mu \nu}=0 .
$$

Since the elements of the matrices $X$ and $M$ are numbers and $\mathcal{V}_{S} \times I$ commutes with $X$ we have from (2.14)

$$
[X, M]=-\hat{H} \cdot \frac{\partial \mathcal{V}}{\partial q}
$$

which is the content of the usual Lax pair.

\section{Spin Calogero-Moser Model with Degenerate Poten- tial}

Now let us define a spin Calogero-Moser model associated with a root system $\Delta$ and an irreducible representation $\mathcal{R}$ of the reflection (Weyl) group $G_{\Delta}$, that is the set of "sites". A dynamical state of the model is a wavefunction $\psi(q)$ times a vector $\psi_{S}$ which takes value in the $D$ multiple of a vector space $\mathbf{V}$;

$$
\psi_{S} \in \stackrel{D}{\otimes} \mathbf{V} .
$$

Each $\mathbf{V}$ is associated with site $\mu$. In other words $\psi_{S}$ can be represented by its component spin $\psi_{S}^{(\mu)}$ at the site $\mu$, or $\psi_{S}^{(j)}$ at site $j$ for short:

$$
\psi_{S}=\mid \psi_{S}^{(1)}, \ldots, \psi_{S}^{(D)}>
$$

Let us introduce a spin exchange operator $\hat{\mathcal{P}}_{\rho}$ associated with each root $\rho \in \Delta$ :

$$
\hat{\mathcal{P}}_{\rho}: \psi_{S} \rightarrow \hat{\mathcal{P}}_{\rho} \psi_{S}, \quad\left(\hat{\mathcal{P}}_{\rho} \psi_{S}\right)^{(\mu)}=\psi_{S}^{\left(s_{\rho}(\mu)\right)}, \quad \forall \mu \in \mathcal{R}
$$


Obviously $\left\{\hat{\mathcal{P}}_{\rho}\right\}(\rho \in \Delta)$ satisfy the same commutation relations as $\left\{\hat{s}_{\rho}\right\}$ :

$$
\hat{\mathcal{P}}_{\alpha} \hat{\mathcal{P}}_{\beta} \hat{\mathcal{P}}_{\alpha}=\hat{\mathcal{P}}_{s_{\alpha}(\beta)}, \quad \hat{\mathcal{P}}_{\alpha}^{2}=1, \quad \hat{\mathcal{P}}_{-\alpha}=\hat{\mathcal{P}}_{\alpha}
$$

and $\hat{s}_{\alpha}, \hat{H}_{j}$ and $\hat{\mathcal{P}}_{\beta}$ commute since they act on different spaces

$$
\left[\hat{s}_{\alpha}, \hat{\mathcal{P}}_{\beta}\right]=0=\left[\hat{H}_{j}, \hat{\mathcal{P}}_{\beta}\right]
$$

Likewise the quantum operators $\left\{q_{j}\right\}$ and $\left\{p_{k}\right\}$ commute with $\hat{\mathcal{P}}_{\rho}$ :

$$
\left[q_{j}, \hat{\mathcal{P}}_{\rho}\right]=0=\left[p_{k}, \hat{\mathcal{P}}_{\rho}\right], \quad j, k=1, \ldots, r, \quad \forall \rho \in \Delta
$$

By multiplying $\hat{\mathcal{P}}_{\rho}$ to the functions $x(\rho \cdot q)$ and $y(\rho \cdot q)$ in $X$ and $\widetilde{M}$, we define new matrices $X_{S}$ and $\widetilde{M}_{S}$ :

$$
\begin{aligned}
X_{S} & =i \sum_{\rho \in \Delta_{+}} g_{|\rho|}(\rho \cdot \hat{H}) x(\rho \cdot q) \hat{\mathcal{P}}_{\rho} \hat{s}_{\rho} \\
\widetilde{M}_{S} & =\frac{i}{2} \sum_{\rho \in \Delta_{+}} g_{|\rho|}|\rho|^{2} y(\rho \cdot q) \hat{\mathcal{P}}_{\rho} \hat{s}_{\rho}
\end{aligned}
$$

whose elements are no longer numbers but operators now. As in the previous section we define a new matrix $M_{S}$,

$$
M_{S}=\widetilde{M}_{S}+i \mathcal{A} \times I
$$

which satisfies sum up to zero condition, too

$$
\sum_{\mu \in \mathcal{R}}\left(M_{S}\right)_{\mu \nu}=\sum_{\nu \in \mathcal{R}}\left(M_{S}\right)_{\mu \nu}=0
$$

The operator $\mathcal{A}$ now depends on the spin exchange operators $\left\{\hat{\mathcal{P}}_{\rho}\right\}$ :

$$
\mathcal{A}=\frac{1}{2} \sum_{\rho \in \Delta_{+}} g_{|\rho|}|\rho|^{2} V(\rho \cdot q) \hat{\mathcal{P}}_{\rho} .
$$

Since the commutation relations of $\left\{\hat{H}_{j}, \hat{s}_{\rho}\right\}$ and $\left\{\hat{H}_{j}, \hat{\mathbf{s}}_{\rho} \equiv \hat{\mathcal{P}}_{\rho} \hat{s}_{\rho}\right\}$ are identical we have the following main result

$$
\left[X_{S}, \widetilde{M}_{S}\right]=-\hat{H} \cdot \frac{\partial \mathcal{V}}{\partial q}
$$

in which the right hand side does not contain operators $\left\{\hat{\mathcal{P}}_{\rho}\right\}$. This is because they cancel out by the relation $\hat{\mathcal{P}}_{\rho}^{2}=1$. The right hand side can be replaced by the obvious identity in quantum theory

$$
-\hat{H} \cdot \frac{\partial \mathcal{V}}{\partial q}=i\left[\mathcal{H}_{C}, p \cdot \hat{H}\right]
$$


If we rewrite $\widetilde{M}_{S}$ in terms of $M_{S}$, we obtain

$$
\left[X_{S}, M_{S}-i \mathcal{A}\right]=i\left[\mathcal{H}_{C}, p \cdot \hat{H}\right]
$$

in which the second commutator in the left hand side no longer vanishes. By adding (3.14) to

$$
\left[p \cdot \hat{H}, M_{S}-i \mathcal{A}\right]=i\left[\frac{p^{2}}{2}, X_{S}\right]
$$

we arrive at the desired equation

$$
\begin{aligned}
{\left[p \cdot \hat{H}+X_{S}, M_{S}\right] } & =i\left[\mathcal{H}_{S}, p \cdot \hat{H}+X_{S}\right] \\
\mathcal{H}_{S} & \equiv \mathcal{H}_{C}-\mathcal{A}=\frac{1}{2} p^{2}+\frac{1}{2} \sum_{\rho \in \Delta_{+}}|\rho|^{2} g_{|\rho|}\left(g_{|\rho|}-\hat{\mathcal{P}}_{\rho}\right) V(\rho \cdot q),
\end{aligned}
$$

which is a universal Lax equation for the spin Calogero-Moser model

$$
i\left[\mathcal{H}_{S}, L_{S}\right]=\left[L_{S}, M_{S}\right], \quad L_{S}=p \cdot \hat{H}+X_{S}
$$

defined by the Hamiltonian $\mathcal{H}_{S}$ (3.17). That is, this applies to any spin Calogero-Moser models based on any root system $\Delta$ and any irreducible representation $\mathcal{R}$ of the reflection (Weyl) group $G_{\Delta}$ and for any degenerate potentials. From this follows

$$
i\left[\mathcal{H}_{S}, L_{S}^{k}\right]=\left[L_{S}^{k}, M_{S}\right], \quad \text { or } \quad i\left[\mathcal{H}_{S},\left(L_{S}^{k}\right)_{\mu \nu}\right]=\sum_{\kappa \in \mathcal{R}}\left\{\left(L_{S}^{k}\right)_{\mu \kappa}\left(M_{S}\right)_{\kappa \nu}-\left(M_{S}\right)_{\mu \kappa}\left(L_{S}^{k}\right)_{\kappa \nu}\right\} .
$$

Thanks to the sum up to zero condition of $M_{S}(3.10)$ we obtain the conserved quantity as the Total sum (Ts) of $L_{S}^{k}$ instead of the diagonal sum (Tr):

$$
\left[\mathcal{H}_{S}, \operatorname{Ts}\left(L_{S}^{k}\right)\right]=0, \quad \operatorname{Ts}\left(L_{S}^{k}\right) \equiv \sum_{\mu, \nu \in \mathcal{R}}\left(L_{S}^{k}\right)_{\mu \nu}, \quad k=2, \ldots,
$$

This type of conserved quantities was known for the $A_{r}$ spin Calogero-Moser models for the vector representation $[7,9$. Note that (3.15) is obtained from 2.15$)$ by replacing $X$ and $\widetilde{M}$ by $X_{S}$ and $\widetilde{M}_{S}$.

Some remarks are in order.

1. When all the spins are the same

$$
\psi_{S}^{(1)}=\psi_{S}^{(2)}=\cdots=\psi_{S}^{(D)}
$$

the action of the spin exchange operators become that of the identity operator

$$
\hat{\mathcal{P}}_{\rho}=1, \quad \forall \rho \in \Delta \text {. }
$$


Then the Hamiltonian $\mathcal{H}_{S}$ (3.17) reduces to that of the quantum Calogero-Moser models and the Lax operator $L_{S}$ and $M_{S}$ become identical to the universal quantum Lax pair operator derived by Bordner, Manton and Sasaki 18 .

2. The form of the spin Calogero-Moser Hamiltonian (3.17) depends on the root system $\Delta$ only, although its actual operator contents depend on the chosen representation $\mathcal{R}$.

3. For the $A_{r}$ model with the vector representation, the present spin Calogero-Moser coincides with the existing one. For the other root systems the present model is completely new, to the best of our knowledge (see the remarks in the following entry). It should be emphasised that even for the $A_{r}$ root system the present formulation of the spin Calogero-Moser models defines an infinitely many different models corresponding to the infinitely many irreducible representations of the symmetric group $\mathcal{S}_{r+1}$, which is the Weyl group of $A_{r}$.

4. For the $A_{r}$ model with the vector representation the number of "sites" is $r+1$ which is equal to the degrees of freedom of the associated particle motion, if the $A_{r}$ root system is embedded into $\mathbf{R}^{r+1}$ as is done customarily. This is a rather exceptional situation. In all the other irreducible representations $\mathcal{S}_{r+1}$ and for all the other root systems (except for the trivial representation), the number of sites, or the dimensions of $\mathcal{R}$, is bigger than $r$, the rank of $\Delta$. For example, the vector representation of $D_{r}$ or the set of short roots for $B_{r}$ consists of $2 r$ vectors, which in a conventional parametrisation of the roots take the form $\mathcal{R}=\left\{ \pm \mathbf{e}_{j}, j=1, \ldots, r \mid \mathbf{e}_{j} \in \mathbf{R}^{r}, \mathbf{e}_{j} \cdot \mathbf{e}_{k}=\delta_{j k}\right\}$. Our spin Calogero-Moser models require all these $2 r$ sites. There are some references in which spin Calogero-Moser models for $B_{r}, C_{r}, D_{r}$ or $B C_{r}$ are discussed [10, 11, 12]. In all these papers, the number of sites is equal to the rank of the root systems. These are different from the present spin Calogero-Moser models.

5. The present formulation of the spin Calogero-Moser models together with the Lax pair formulation does not require any specific structure of the "spin" space V attached to each site.

6. It is well-known that for the spin $1 / 2$ case in the $A_{r}$ model with the vector representation, the spin exchange operators $\left\{\hat{\mathcal{P}}_{\rho}\right\}$ can be expressed in terms of the local Pauli spin matrix at each site as $\hat{\mathcal{P}}_{\mathbf{e}_{j}-\mathbf{e}_{k}}=\left(1+\vec{\sigma}_{j} \cdot \vec{\sigma}_{k}\right) / 2$. For the vector representation of 
$D_{r}$ or $\mathcal{R}$ being the set of short roots for $B_{r}$ mentioned above, we have

$$
\begin{array}{ll}
\hat{\mathcal{P}}_{\mathbf{e}_{j}}=\left[\left(1+\vec{\sigma}_{j} \cdot \vec{\sigma}_{-j}\right) / 2\right], & \hat{\mathcal{P}}_{\mathbf{e}_{j}-\mathbf{e}_{k}}=\left[\left(1+\vec{\sigma}_{j} \cdot \vec{\sigma}_{k}\right) / 2\right]\left[\left(1+\vec{\sigma}_{-j} \cdot \vec{\sigma}_{-k}\right) / 2\right], \\
& \hat{\mathcal{P}}_{\mathbf{e}_{j}+\mathbf{e}_{k}}=\left[\left(1+\vec{\sigma}_{j} \cdot \vec{\sigma}_{-k}\right) / 2\right]\left[\left(1+\vec{\sigma}_{-j} \cdot \vec{\sigma}_{k}\right) / 2\right] .
\end{array}
$$

In other words, $\hat{\mathcal{P}}_{\mathbf{e}_{j}+\mathbf{e}_{k}}$ exchanges the spins at site $j$ and $-k$ and simultaneously the spins at $-j$ and $k$. Similar expressions exist for other representations and root systems for $s u(2), s u(N)$ or other spins.

7. It is easy to verify, as in the Calogero-Moser models, that the Hamiltonian $\mathcal{H}_{S}$ (3.17) is obtained as the lowest member of the conserved quantities derived from the Lax pair formulation:

$$
\mathcal{H}_{S} \propto \operatorname{Ts}\left(L_{S}^{2}\right)
$$

8. The conserved quantities $\left\{\operatorname{Ts}\left(L_{S}^{k}\right)\right\}$ are essentially the same as those obtained in terms of the Dunkl [20] operators, and/or the exchange operator formalism [8]. The same remark applies to the conserved quantities of the spin exchange models to be discussed in the following section. For the quantum CM models without spin, the equivalence of the Lax pair formalism and Dunkl operator formalism was proven in [19].

9. The Yangian symmetry [21, 22] for the spin CM model and spin exchange model based on any root system is an interesting challenge.

10. The commutativity of the conserved quantities obtained from the above Lax pair formulation will be discussed elsewhere.

\subsection{Rational Spin Calogero Model}

In this subsection we will define rational spin Calogero-Moser model with quadratic confining potential to be called rational spin Calogero model, for brevity. The Hamiltonian is given by

$$
\mathcal{H}_{R S}=\frac{1}{2} p^{2}+\frac{1}{2} \omega^{2} q^{2}+\frac{1}{2} \sum_{\rho \in \Delta_{+}} \frac{|\rho|^{2} g_{|\rho|}\left(g_{|\rho|}-\hat{\mathcal{P}}_{\rho}\right)}{(\rho \cdot q)^{2}} .
$$

The construction of the Lax pair follows the same pattern as the case without the spin degrees of freedom. Since the added potential $\frac{1}{2} \omega^{2} q^{2}$ commutes with $X_{S}$, the canonical equations of motion to be obtained from $\mathcal{H}_{R S}$ are equivalent to

$$
\dot{L}_{S}=i\left[\mathcal{H}_{R S}, L_{S}\right]=\left[L_{S}, M_{S}\right]-\omega^{2} Q, \quad Q \equiv q \cdot \hat{H},
$$


in which $L_{S}$ and $M_{S}$ are the Lax pair for the rational $\left(1 /(\rho \cdot q)^{2}\right)$ potential only. Let us define

$$
L_{S}^{ \pm}=L_{S} \pm i \omega Q
$$

whose time evolution read

$$
\dot{L}_{S}^{ \pm}=\left[L_{S}^{ \pm}, M_{S}\right] \pm i \omega L^{ \pm} .
$$

Here we have used well-known relations [17, 18]

$$
\dot{Q}=p \cdot \hat{H}=L_{S}-X_{S}, \quad\left[Q, M_{S}\right]=-X_{S} .
$$

If we define

$$
\mathcal{L}_{S}=L_{S}^{+} L_{S}^{-},
$$

its time evolution is Lax like:

$$
\dot{\mathcal{L}}_{S}=i\left[\mathcal{H}_{R S}, \mathcal{L}_{S}\right]=\left[\mathcal{L}_{S}, M_{S}\right]
$$

Thus we obtain conserved quantities

$$
\operatorname{Ts}\left(\mathcal{L}^{k}\right), \quad k=1, \ldots
$$

The lowest conserved quantity $\operatorname{Ts}(\mathcal{L})$ gives the Hamiltonian $\mathcal{H}_{R S}(3.23)$

$$
\operatorname{Ts}(\mathcal{L}) \propto \mathcal{H}_{R S}+\left(\frac{r}{2}+\sum_{\rho \in \Delta_{+}} g_{|\rho|} \hat{\mathcal{P}}_{\rho}\right),
$$

plus additional terms which commute with all the spin exchange operators $\left\{\hat{\mathcal{P}}_{\rho}\right\}$.

\section{Spin Exchange Model}

The spin exchange model is defined for a root system $\Delta$ and an irreducible representation $\mathcal{R}$ of the reflection (Weyl) group $G_{\Delta}$. Its dynamical state is represented by a vector $\psi_{S}$ only which takes value in the $D$ multiple of a vector space $\mathbf{V}$;

$$
\psi_{S} \in \stackrel{D}{\otimes} \mathbf{V} .
$$

As in the spin Calogero-Moser model case each $\mathbf{V}$ is associated with site $\mu$. In other words $\psi_{S}$ can be represented by its component spin $\psi_{S}^{(\mu)}$ at the site $\mu$, or $\psi_{S}^{(j)}$ at site $j$ for short:

$$
\psi_{S}=\mid \psi_{S}^{(1)}, \ldots, \psi_{S}^{(D)}>.
$$


In fact, the spin exchange model is obtained from the corresponding spin Calogero-Moser model by "freezing" the particle degrees of freedom:

$$
p=0, \quad q=q_{0},
$$

in which $q_{0}$ is an equilibrium position of the classical Calogero-Moser potential

$$
\left.\frac{\partial \mathcal{V}}{\partial q}\right|_{q=q_{0}}=0, \quad \mathcal{V}=\frac{1}{2} \sum_{\rho \in \Delta_{+}} g_{|\rho|}^{2}|\rho|^{2} V(\rho \cdot q) .
$$

Since the rational potential without the quadratic confining potential or the hyperbolic potential do not have any equilibrium points, this automatically selects the trigonometric potential. The rational potential with the quadratic confining potential case will be discussed in the next subsection separately. The equilibrium position $q_{0}$ for the trigonometric potential is determined uniquely in each Weyl alcove. In other words, if $q_{0}$ is an equilibrium point so is $s_{\alpha}\left(q_{0}\right)$ which defines an equally integrable model. Let us fix $q_{0}$ and define $X_{E}$ and $\widetilde{M}_{E}$ in terms of the Lax pair operators of the corresponding spin Calogero-Moser model at $q=q_{0}$ :

$$
X_{E}=\left.X_{S}\right|_{q=q_{0}}, \quad \widetilde{M}_{E}=\left.\widetilde{M}_{S}\right|_{q=q_{0}} .
$$

The components of the matrices $X_{E}$ and $\widetilde{M}_{E}$ are linear combinations of the spin exchange operators $\hat{\mathcal{P}}_{\rho}$ and the coefficients are just numbers. They satisfy a simple matrix identity

$$
\left[X_{E}, \widetilde{M}_{E}\right]=0
$$

and as before $\widetilde{M}_{E}$ has a special property:

$$
\sum_{\mu \in \mathcal{R}}\left(\widetilde{M}_{E}\right)_{\mu \nu}=\sum_{\nu \in \mathcal{R}}\left(\widetilde{M}_{E}\right)_{\mu \nu}=-i \mathcal{A}_{E}, \quad \mathcal{A}_{E}=\frac{1}{2} \sum_{\rho \in \Delta_{+}} g_{|\rho|}|\rho|^{2} V\left(\rho \cdot q_{0}\right) \hat{\mathcal{P}}_{\rho},
$$

As in the previous section we define a new matrix $M_{E}$,

$$
M_{E}=\widetilde{M}_{E}+i \mathcal{A}_{E} \times I,
$$

which satisfies sum up to zero condition, too

$$
\sum_{\mu \in \mathcal{R}}\left(M_{E}\right)_{\mu \nu}=\sum_{\nu \in \mathcal{R}}\left(M_{E}\right)_{\mu \nu}=0 .
$$

By rewriting (4.5) in terms of $M_{E}$ we arrive at the Lax representation of the spin exchange model:

$$
i\left[\mathcal{H}_{E}, X_{E}\right]=\left[X_{E}, M_{E}\right],
$$


in which the Hamiltonian $\mathcal{H}_{E}$ of the spin exchange model is

$$
\mathcal{H}_{E}=\frac{1}{2} \sum_{\rho \in \Delta_{+}} g_{|\rho|}|\rho|^{2} V\left(\rho \cdot q_{0}\right)\left(1-\hat{\mathcal{P}}_{\rho}\right)=-\mathcal{A}_{E}+\text { const }
$$

The added constant simply shifts the ground state energy. The Lax pair supplies the conserved quantities as the Total sum of $X_{E}^{k}$ :

$$
\left[\mathcal{H}_{E}, \operatorname{Ts}\left(X_{E}^{k}\right)\right]=0, \quad \operatorname{Ts}\left(X_{E}^{k}\right) \equiv \sum_{\mu, \nu \in \mathcal{R}}\left(X_{E}^{k}\right)_{\mu \nu}, \quad k=3, \ldots,
$$

It is interesting to note that the first two members $\operatorname{Ts}\left(X_{E}^{1}\right)$ and $\operatorname{Ts}\left(X_{E}^{2}\right)$ are trivial, in contrast to the spin Calogero-Moser case.

Some remarks are in order.

1. As in the spin Calogero-Moser model, the form of the spin exchange model Hamiltonian $\mathcal{H}_{E}$ (4.8) depends on the root system $\Delta$ only, although its actual operator contents depend on the chosen representation $\mathcal{R}$. The infinitely many models corresponding to various irreducible representations, sharing the same set of conserved quantities, can be considered to constitute an integrable hierarchy belonging to the root system $\Delta$. If one considers a series of representations with increasing dimensionality (i.e. more spins), the thermodynamic limit could be achieved within models belonging to a fixed root system $\Delta$. This is a novel situation, since in the Haldane-Shastry model the rank $r$ grows indefinitely in the thermodynamic limit.

2. It should be remarked that the $q_{0}$ is the equilibrium point not of the function appearing in the Hamiltonian $\mathcal{H}_{E}$ (4.8) which is linear in the coupling constants $g_{|\rho|}$ but that of the potential of the classical Calogero-Moser Hamiltonian $\mathcal{H}_{C}(2.3)$ which is quadratic in the coupling constants. This difference is meaningful only for the models based on non-simply laced root systems.

3. It should be emphasised that the "coordinates" $q$ or rather $q_{0}$ are just a set of numbers rather than dynamical variables. Thus, in contrast to the conventional approach [5, 8], the notion of 'position exchange operator' is not used in our approach.

4. For the $A_{r}$ model, $q_{0}$ can be chosen to be "equidistant":

$$
q_{0}=\pi(1,2, \ldots, r, r+1) /(r+1) \text {, }
$$


thanks to the well-known trigonometric identity

$$
\sum_{k \neq j}^{r+1} \frac{\cos [\pi(j-k) /(r+1)]}{\sin ^{3}[\pi(j-k) /(r+1)]}=0
$$

The Haldane-Shastry model [5], i.e. the $A_{r}$ spin exchange model for the vector representation, has been understood quite well because of this simplifying feature.

5. The equidistance of $q_{0}$ for $A_{r}$ seems rather fortuitous. As remarked above, any transposition of the above $q_{0}(4.10)$ provides an equally integrable spin exchange model, but the equidistance property is lost. As for $D_{r}(r \geq 4)$, we have not been able to find equidistant $q_{0}$. For $B C_{r}$ model, equidistant $q_{0}$ can be achieved for certain ratios of the coupling constants. For the following parametrisation of the potential [4, 16],

$$
\mathcal{V}=\sum_{j<k}^{r}\left[\frac{g_{M}^{2}}{\sin ^{2}\left(q_{j}-q_{k}\right)}+\frac{g_{M}^{2}}{\sin ^{2}\left(q_{j}+q_{k}\right)}\right]+\sum_{j=1}^{r} \frac{g_{S}\left(g_{S}+2 g_{L}\right)}{2 \sin ^{2}\left(q_{j}\right)}+\sum_{j=1}^{r} \frac{2 g_{L}^{2}}{\sin ^{2}\left(2 q_{j}\right)},
$$

one obtains equidistant equilibrium positions:

$$
\begin{aligned}
& q_{0}=\pi(1,3, \ldots, 2 r-1) / 4 r, \quad \text { for } \quad g_{L} / g_{M}=1 / 2, \quad g_{S}=0, \\
& q_{0}=\pi(1,2, \ldots, r) / 2(r+1), \quad \text { for } \quad g_{L} / g_{M}=3 / 2, \quad g_{S}=0 \\
& q_{0}=\pi(1,2, \ldots, r) /(2 r+1), \quad \text { for } \quad g_{L} / g_{M}=1 / 2, \quad g_{S} / g_{M}=1 .
\end{aligned}
$$

These cases were discussed in some detail by Bernard-Pasquier-Serban [1].

6. Note that the present derivation of the spin exchange model and its Lax pair does not adopt the strong coupling limit.

7. For most general elliptic potentials, the Lax pair can be constructed in a usual manner [14]. But the second Lax operator does not satisfy the "sum to zero" condition, hence the integrability of these models is not yet established.

\subsection{Rational Spin Exchange Model}

The above formulation fails to give integrable spin exchange model with rational potential. This can be remedied by adding a harmonic confining potential [8, 12] which creates equilibrium points in each Weyl chamber. Here we derive the Lax operator formalism for these

models. Let us start with the Lax pair for the rational Calogero-Moser models and for the time being keep the value of $q$ unspecified. We have as in (2.14)

$$
[X, \widetilde{M}]=-\hat{H} \cdot \frac{\partial \mathcal{V}}{\partial q}
$$


and after multiplying $\hat{\mathcal{P}}_{\rho}$ to functions $x(\rho \cdot q)$ and $y(\rho \cdot q)$, we obtain (3.12)

$$
\left[X_{S}, \widetilde{M}_{S}\right]=-\hat{H} \cdot \frac{\partial \mathcal{V}}{\partial q} \text {. }
$$

The diagonal matrix $Q(\sqrt{3.19})$ satisfies the relation $(3.27)$

$$
\left[Q, \widetilde{M}_{S}\right]=-X_{S}
$$

If we define two new matrices $X_{S}^{ \pm}$

$$
X_{S}^{ \pm}=X_{S} \pm i \omega Q
$$

they satisfy simple commutation relations thanks to (4.15) and 4.16)

$$
\left[X_{S}^{ \pm}, \widetilde{M}_{S}\right]=\mp i \omega X_{S}^{ \pm}-\hat{H} \cdot \frac{\partial \mathcal{V}_{R C}}{\partial q}
$$

in which $\mathcal{V}_{R C}$ is the potential of the classical rational Calogero-Moser model with harmonic confining potential

$$
\mathcal{V}_{R C}=\frac{1}{2} \omega^{2} q^{2}+\frac{1}{2} \sum_{\rho \in \Delta_{+}} \frac{g_{|\rho|}^{2}|\rho|^{2}}{(\rho \cdot q)^{2}} .
$$

Now we choose $q_{0}$ as an equilibrium point of $\mathcal{V}_{R C}$ and define

$$
X_{R E}^{ \pm}=\left.X_{S}^{ \pm}\right|_{q=q_{0}}, \quad \widetilde{M}_{R E}=\left.\widetilde{M}_{S}\right|_{q=q_{0}},\left.\quad \frac{\partial \mathcal{V}_{R C}}{\partial q}\right|_{q=q_{0}}=0
$$

Thus we arrive at

$$
\left[X_{R E}^{+} X_{R E}^{-}, \widetilde{M}_{R E}\right]=X_{R E}^{+}\left[X_{R E}^{-}, \widetilde{M}_{R E}\right]+\left[X_{R E}^{+}, \widetilde{M}_{R E}\right] X_{R E}^{-}=0
$$

We define $M_{R E}$ by

$$
\begin{aligned}
M_{R E} & =\widetilde{M}_{R E}+i \mathcal{A}_{R E} \times I, \\
\mathcal{A}_{R E} & =\frac{1}{2} \sum_{\rho \in \Delta_{+}} \frac{g_{|\rho|}|\rho|^{2} \hat{\mathcal{P}}_{\rho}}{\left(\rho \cdot q_{0}\right)^{2}},
\end{aligned}
$$

so that $M_{R E}$ satisfies the sum to zero condition

$$
\sum_{\mu \in \mathcal{R}}\left(M_{R E}\right)_{\mu \nu}=\sum_{\nu \in \mathcal{R}}\left(M_{R E}\right)_{\mu \nu}=0
$$

Then (4.21) can be rewritten as a Lax representation for the rational spin exchange model

$$
i\left[\mathcal{H}_{R E}, X_{R E}^{+} X_{R E}^{-}\right]=\left[X_{R E}^{+} X_{R E}^{-}, M_{R E}\right]
$$


in which the rational spin exchange Hamiltonian $\mathcal{H}_{R E}$ is defined by

$$
\mathcal{H}_{R E}=\frac{1}{2} \sum_{\rho \in \Delta_{+}} \frac{g_{|\rho|}|\rho|^{2}}{\left(\rho \cdot q_{0}\right)^{2}}\left(1-\hat{\mathcal{P}}_{\rho}\right)-\mathcal{A}_{R E}+\text { const } .
$$

The conserved quantities are obtained as Total sum of $\left(X_{R E}^{+} X_{R E}^{-}\right)^{k}$ :

$$
\left[\mathcal{H}_{R E}, \operatorname{Ts}\left(\left(X_{R E}^{+} X_{R E}^{-}\right)^{k}\right)\right]=0, \quad k=1, \ldots, .
$$

It is interesting to note that the above Hamiltonian $\mathcal{H}_{R E}$ depends on the harmonic confining potential $\frac{1}{2} \omega^{2} q^{2}$ only through the value $q_{0}$.

\section{Summary and comments}

We have shown that the integrability of spin Calogero-Moser model and the spin exchange model with degenerate potential and based on any root system is a direct consequence of the integrability of the corresponding classical Calogero-Moser system. For a given root system $\Delta$ there are infinitely many integrable spin Calogero-Moser models and the spin exchange models corresponding to infinitely many irreducible representations $\mathcal{R}$ of the reflection group. These define physically different models sharing the same exchange features.

After completion of the present work, we came across 23 which discusses the integrability of spin $B C_{r}$ model with harmonic confining potential, or "spin Inozemtsev model" [4] in terms of the Dunkl operator formalism [20].

\section{Acknowledgements}

We thank D. B. Fairlie for bringing [23] to our attention. V. I. I. is supported by JSPS long term fellowship. R. S. is partially supported by the Grant-in-aid from the Ministry of Education, Culture, Sports, Science and Technology, Japan, priority area (\#707) "Supersymmetry and unified theory of elementary particles".

\section{References}

[1] F. Calogero, "Solution of the one-dimensional $N$-body problem with quadratic and/or inversely quadratic pair potentials", J. Math. Phys. 12 (1971) 419-436.

[2] B. Sutherland, "Exact results for a quantum many-body problem in one-dimension. II", Phys. Rev. A5 (1972) 1372-1376. 
[3] J. Moser, "Three integrable Hamiltonian systems connected with isospectral deformations", Adv. Math. 16 (1975) 197-220; J. Moser, "Integrable systems of non-linear evolution equations", in Dynamical Systems, Theory and Applications; J. Moser, ed., Lecture Notes in Physics 38 (1975), Springer-Verlag; F. Calogero, C. Marchioro and O. Ragnisco, "Exact solution of the classical and quantal one-dimensional many body problems with the two body potential $V_{a}(x)=g^{2} a^{2} / \sinh ^{2} a x "$, Lett. Nuovo Cim. 13 (1975) 383-387; F. Calogero, "Exactly solvable one-dimensional many body problems", Lett. Nuovo Cim. 13 (1975) 411-416.

[4] V.I. Inozemtsev and D. V. Meshcheryakov, "Extension of the class of integrable dynamical systems connected with semisimple Lie algebras", Lett. Math. Phys. 9 (1985) 13-18; V.I. Inozemtsev, "Lax representation with spectral parameter on a torus for integrable particle systems", Lett. Math. Phys. 17 (1989) 11-17.

[5] F. D. M. Haldane, "Exact Jastrow-Gutzwiller resonating valence bond ground state of the spin 1/2 antiferromagnetic Heisenberg chain with $1 / \mathrm{r}^{* *} 2$ exchange", Phys. Rev. Lett. 60 (1988) 635-638; B. S. Shastry, "Exact solution of $S=1 / 2$ Heisenberg antiferromagnetic chain with long-ranged interactions", ibid 60 (1988) 639-642.

[6] V. I. Inozemtsev, "On the connection between the one-dimensional $S=1 / 2$ Heisenberg chain and Haldane-Shastry model", J. Stat. Phys. 59 (1990) 1143-1156.

[7] B. S. Shastry and B. Sutherland, "Superlax pairs and infinite symmetries in the $1 / r^{2}$ system", Phys. Rev. Lett. 70 (1993) 4029-4033; B. Sutherland and B. S. Shastry, "Solutions of some integrable one-dimensional quantum system", Phys. Rev. Lett. 71 (1993) $5-8$.

[8] A.P. Polychronakos, "Exchange operator formalism for integrable systems of particles", Phys. Rev. Lett. 69 (1992) 703-705; M. Fowler and J.A. Minahan, "Invariants of the Haldane-Shastry $S U(N)$ chain", Phys. Rev. Lett. 70 (1993) 2325-2328; A.P.Polychronakos, "Lattice integrable systems of Haldane-Shastry type", ibid $\mathbf{7 0}$ (1993) 2329-2331.

[9] K. Hikami and M. Wadati, "Integrability of Calogero-Moser spin system", J. Phys. Soc. Jpn. 62 (1993) 469-472. 
[10] B. D. Simons and B. L. Altschuler, "Exact ground state of an open $S=1 / 2$ long-range Heisenberg antiferromagnetic spin chain", Phys. Rev. B50 (1994) 1102-1105.

[11] D. Bernard, V. Pasquier and D. Serban, "Exact solution of long-range interacting spin chains with boundaries", Europhys. Lett. 30 (1995) 301-306.

[12] T. Yamamoto, "Multicomponent Calogero model of $B_{N}$-type confined in a harmonic potential", Phys. Lett. A208 (1995) 293; T. Yamamoto and O. Tsuchiya, "Integrable 1/r ${ }^{2}$ spin chain with reflecting end", J. Phys. A29 (1996) 3977-3984, cond-mat/9602105.

[13] V. I. Inozemtsev, "Invariants of linear combinations of transpositions", Lett. Math. Phys. 36 (1996) 55-63.

[14] M. A. Olshanetsky and A. M. Perelomov, "Completely integrable Hamiltonian systems connected with semisimple Lie algebras", Inventions Math. 37 (1976), 93-108; "Classical integrable finite-dimensional systems related to Lie algebras", Phys. Rep. C71 (1981), 314-400.

[15] E. D'Hoker and D.H.Phong, “Calogero-Moser Lax pairs with spectral parameter for general Lie algebras", Nucl. Phys. B530 (1998) 537-610, hep-th/9804124.

[16] A. J. Bordner, E. Corrigan and R. Sasaki, "Calogero-Moser models I: a new formulation", Prog. Theor. Phys. 100 (1998) 1107-1129, hep-th/9805106; A. J. Bordner, R. Sasaki and K. Takasaki, "Calogero-Moser models II: symmetries and foldings", Prog. Theor. Phys. 101 (1999) 487-518, hep-th/9809068; A. J. Bordner and R. Sasaki, "Calogero-Moser models III: elliptic potentials and twisting", Prog. Theor. Phys. 101 (1999) 799-829, hep-th/9812232; S. P. Khastgir, R. Sasaki and K. Takasaki, "Calogero-Moser models IV: Limits to Toda theory", Prog. Theor. Phys. 102 (1999), 749-776, hep-th/9907102.

[17] A. J. Bordner, E. Corrigan and R. Sasaki, "Generalized Calogero-Moser models and universal Lax pair operators", Prog. Theor. Phys. 102 (1999) 499-529, hep-th/9905011.

[18] A. J. Bordner, N. S. Manton and R. Sasaki, "Calogero-Moser models V: Supersymmetry, and Quantum Lax Pair", Prog. Theor. Phys. 103 (2000) 463-487, hep-th/9910033.

[19] S. P. Khastgir, A. J. Pocklington and R. Sasaki, "Quantum Calogero-Moser Models: Integrability for all Root Systems", J. Phys. A33 (2000) 9033-9064, hep-th/0005277. 
[20] C.F. Dunkl, "Differential-difference operators associated to reflection groups", Trans. Amer. Math. Soc. 311 (1989) 167-183; "Orthogonal polynomials of types $A$ and $B$ and related Calogero models", Comm. Math. Phys. 197 (1998) 451-487.

[21] F. D. M. Haldane, Z. N. C. Ha, J. C. Talstra, D. Bernard and V. Pasquier, "Yangian symmetry of integrable quantum chains with long-range interactions and a new description of states in conformal field theory", Phys. Rev. Lett. 69 (1992) 2021-2025; Yang-Baxter equation in long-range interacting systems", J. Phys. A26 (1993) 5219-5236.

[22] K. Hikami, "Yangian symmetry and Virasoro character in a 1 attice spin system with long-range interactions", Nucl. Phys. B441 (1995) 530-548.

[23] F. Finkel, D. Gomez-Ullate, A. Gonzalez-Lopez, M.A. Rodriguez, R. Zhdanov, " $A_{N^{-}}$ type Dunkl operators and new spin Calogero-Sutherland models", hep-th/0102039, February 2001; "New spin Calogero-Sutherland models related to $B_{N}$-type Dunkl operators", hep-th/0103190, March 2001. 